\title{
Selection of Highly Susceptible Cell Lines to Foot and Mouth Disease Virus Infection
}

\author{
O. Zabal ${ }^{1,2 *}$, N. Fondevila ${ }^{1,2}$ \\ ${ }^{1}$ Instituto de Virologia, CICVyA, INTA, Dr. Nicolas Repetto y De los Reseros s/n, Hurlingham, Argentina \\ ${ }^{2}$ Universidad del Salvador, Cátedra de Enfermedades Infecciosas, Carrera de Veterinária, Pilar, Argentina \\ Email: *ozabal@cnia.inta.gov.ar
}

Received December 19, 2012; revised February 5, 2013; accepted March 5, 2013

Copyright (C) 2013 O. Zabal, N. Fondevila. This is an open access article distributed under the Creative Commons Attribution License, which permits unrestricted use, distribution, and reproduction in any medium, provided the original work is properly cited.

\begin{abstract}
Different non-established cultures were examined to find those that showed high sensitivity to Foot and Mouth Disease Virus (FMDV). Ovine kidney cultures showed high sensitivity to types A, O and C, and were suitable to detect viral infection in samples of animal, as well as cell culture origin. The level of detection in this system was up to ten times higher than in $\mathrm{BHK}_{21}$ cells, which were commonly used for FMDV isolation and production. Viral production levels in ovine kidney cultures ranged from similar to twice as high as in $\mathrm{BHK}_{21}$. Ovine kidney cultures maintained these characteristics for at least 18 passages, allowing their use as an alternative system for FMDV diagnoses.
\end{abstract}

Keywords: Foot-and-Mouth Disease; Tissue Culture; Virus Isolation

\section{Introduction}

Foot-and-mouth disease virus (FMDV) belongs to the family Picornaviridae, Aphtovirus genus, and presents seven serotypes and more than sixty subtypes. The virus causes a highly contagious disease that affects a wide range of domestic and wild animal species. This, combined with the high variability of the virus, determines that control of FMDV infections is challenging and extremely expensive.

In an FMDV outbreak, fast and reliable identification of the etiological agent is essential even in those animals that do not present clinical symptoms. Several diagnostic methods are currently available based on the detection of antigens, nucleic acids or viral infectivity, but given the importance of FMDV, these diagnostic methodologies are generally applied concurrently to increase the chance of detection. Although slower than other methodologies, virus isolation is irreplaceable, not only due to its high sensitivity, but also because it provides viral material for further studies [1].

At present, susceptible cultures normally used for FMDV isolation include $\mathrm{BHK}_{21}$ and IBRS-2 cell lines, as well as bovine, ovine and porcine kidney primary cultures [1]. These tissue cultures can present drawbacks that negatively influence the sensitivity of the detection. On one hand, different clones, passages and manipulation

"Corresponding author. methods of the cell lines have shown up to $2 \log$ differences in sensitivity in inter-laboratory determinations [25]. Also, the potential infection with adventitious virus is a worth-mentioning pitfall of IBRS-2 cells [3]. Primary cultures, on the other hand, are time-consuming, and can rapidly lose sensitivity during successive passages [4].

For these reasons, this work has analyzed different non-established cell lines originated from ovine and bovine primary cultures, to identify those with high and long-lasting sensitivity to FMDV infection.

\section{Materials and Methods}

Cells. Organ slices of bovine fetal thyroid (BTY), bovine fetal tongue epithelium (BTE) and bovine fetal kidney (BFK) from a 6-month-old calf fetus; and ovine kidney (OVK) from a one-day-old lamb were enzymatically disrupted with $0.3 \%(\mathrm{w} / \mathrm{v})$ trypsin and cultivated in Dulbecco's Modified Eagle Medium (DMEM, Gibco) with $10 \%(\mathrm{v} / \mathrm{v})$ fetal bovine serum (FBS), $100 \mathrm{UI} / \mathrm{ml}$ penicillin, $66 \mu \mathrm{g} / \mathrm{ml}$ streptomycin and $50 \mu \mathrm{g} / \mathrm{ml}$ gentamycin (Gibco). Every five passages of the non-established cell lines, 30 vials with $10^{6}$ cells each were frozen in liquid nitrogen to conform a cell bank. All cultures were monitored to rule out contamination of 1) bacteria and fungi, by subculturing in Agar-Sabourad and BHI medium, respectively; 2) mycoplasms, by PCR; and 3) adventitious virus, by cell culture, immunofluorescence and PCR. $\mathrm{BHK}_{21}$ monolay- 
ers, passage 88 - 100 (Asociacion Banco Argentino de Celulas. Buenos Aires, Argentina), amplified in the same medium, were used as control.

Viruses. Samples of serotypes O1C, A2001 and C3 Indaial of FMDV were obtained from the Argentinian Animal Health Service Reference Laboratory collection. For the different assays, two kinds of virus were used: tissue culture-adapted (isolates with four to five passages on $\mathrm{BHK}_{21}$ ), or a sample of oesophagopharyngeal fluid (OPF) of a bovine infected with A2001 virus.

Virus Isolation Assays. In order to determine the sensitivity of the cells to FMDV, comparative titrations were performed. Infectivity measurements were carried out in triplicates in monolayers grown in 24- or 96-well plates (Greiner Bio One) in DMEM supplemented with 2\% fetal calf serum and antibiotics. Sample positivity was determined by observation of cytophathic effects (CPE). The tissue culture infection dose 50 (TCID50) was calculated by the method of Reed and Muench [6], and the means expressed in decimal logarithmic units/ml. Experiments involving FMDV were carried out at Level 3 biosafety facilities (Institute of Virology, INTA, Castelar, Argentina).

To determine positivity percentages, $0.1 \mathrm{ml}$ of a suspension of virus with $10 \log _{10}$ TCID50/ml was inoculated in 48 wells of 96-well plates for each tissue. After $72 \mathrm{~h}$, the percentage of wells with CPE was recorded.

Virus Production. This was evaluated in OVK and $\mathrm{BHK}_{21}$ monolayers grown in 24-well plates. Each well was exposed to a viral inoculum (A2001, O1C, C3 Indaial) with a multiplicity of infection of 0.1 to 0.01 for 30 min, after which $0.9 \mathrm{ml}$ DMEM medium with $2 \%$ FBS was added. After 24 and $48 \mathrm{~h}$ incubation at $37^{\circ} \mathrm{C}$, the virus was titrated in culture supernatants.

Reproducibility. Triplicate titrations of A24, O1C and C3 Indaial virus were carried out in three passages on OVK $(14,15$ and 16), and in three different days (27 replicas).

Statistical Analysis. Comparison of titers obtained in different tissue cultures was done using Student's t-Test. The statistical significance between percentages was estimated by confidence intervals. Reproducibility of results obtained for different passages, days and repetitions was analyzed by nested ANOVA.

\section{Results and Discussion}

A fast and accurate diagnosis is essential in FMDV in order to execute effective control measures. The high variability of the virus can negatively affect the results obtained with all current diagnostic methods, thus a multiple approach might enable a more accurate diagnosis. Viral isolation is very important not only to identify and characterize the virus, but also to amplify live virus from original tissue samples suitable for subsequent studies.
To select the most susceptible cultures, comparative FMDV titrations were carried out during the first cell line passages. Tissue culture-adapted FMDV (O1C with four passages on $\mathrm{BHK}_{21}$ ) was detected by all cultures, but only $\mathrm{OVK}$ and $\mathrm{BFK}$ presented similar titers than $\mathrm{BHK}_{21}$ (7.25, 6.6, 6.74 TCID50/ml, for OVK, BFK and $\mathrm{BHK}_{21}$, respectively), while the titers obtained with the other lines were at least one $\log 10$ lower than with $\mathrm{BHK}_{21}$ (5.39 and 5.6 TCID50/ml, for BTL and BTY, respectively). On the other hand, a sample of oesophagopharyngeal fluid (OPF) of a bovine infected with A2001 virus could only be detected in $\mathrm{BHK}_{21}$, OVK and BFK (2.86, 2.60 and $2.50 \mathrm{TCID} 50 / \mathrm{ml}$, respectively).

Given these results, subsequent studies were designed to identify the most susceptible line between OVK and $\mathrm{BFK}$, by titration with cell culture-adapted $\mathrm{O} 1 \mathrm{C}$ virus and A2001 virus from infected OPF fluids. O1C titers in OVK were significantly higher (Student's T test, $\mathrm{p}<0.05$ ) than those in BFK (7.42 vs 5.75 TCID50/ml, respectively). Additionally, there was a significantly higher percentage of positive wells in OVK than in BFK exposed to virus of infected animals $(13 \%$ vs $0 \%, \mathrm{p}<0.05)$.

Finally, comparative studies between OVK (passages 5 to 18 ) and $\mathrm{BHK}_{21}$ cells were performed. In this step, three FMDV subtypes were used (A2001, C3 Indaial, O1 Campos) and the following parameters were evaluated: viral titers, percentages of positive cultures using 10 TCID50/ml, virus production and reproducibility. Titration of different isolates of the three subtypes showed an array of values that ranged from similar levels between OVK and BHK to ten times higher in OVK. Additionally, OVK maintained or even improved its susceptibility upon successive passages until at least passage 18 (Table 1).

OVK and $\mathrm{BHK}_{21}$ cells, grown in 96-well microtiter plates, inoculated with $100 \mu \mathrm{l}$ of a $10 \mathrm{TCID} 50 / \mathrm{ml}$ suspension of the different viral subtypes, presented similar or higher positive CPE percentages (Table 2). It is noteworthy mentioning that the current control strategy of

Table 1. Titration of different FMDV isolates of the A2001, C3 Indaial and O1C subtypes in BHK21 and OVK cells (passage 5 to 18). Results are expressed as TCID50/ml.

\begin{tabular}{cccccc}
\hline \multicolumn{2}{c}{$\mathrm{A} 2001$} & \multicolumn{2}{c}{$\mathrm{C} 3 \mathrm{I}$} & \multicolumn{2}{c}{$\mathrm{O} 1 \mathrm{C}$} \\
\hline $\mathrm{BHK}_{21}$ & OVK & BHK $_{21}$ & OVK & BHK $_{21}$ & OVK \\
\hline 2.6 & 2.39 & 6.15 & 6.5 & 7.5 & 7.5 \\
5.5 & 5.3 & 5.75 & 6.64 & 4.66 & 5 \\
5.33 & 5.3 & 6.5 & 7.54 & 6 & 6.5 \\
6.5 & 6.5 & 6.4 & 7.85 & 5.66 & 6.5 \\
6 & 6.5 & & & 5.63 & 6.6 \\
6 & 6.5 & & & 6.5 & 7.54 \\
$\leq 0.5$ & 0.6 & & & 6.4 & 7.85
\end{tabular}


FMD in Argentina, which has a disease-free status, consists in the vaccination of all bovines north of latitude 42 . Thus, exposure of an animal to the virus might yield mild infections with low titers, in the range of those observed in this work in the assays of virus isolation in OPF fluid or final dilution.

Virus production was evaluated in $\mathrm{OVK}$ and $\mathrm{BHK}_{21}$ monolayers grown in 24-well plates. Each well was exposed to a viral inoculum with a multiplicity of infection of 0.1 to 0.01 (A2001, O1C, C3 Indaial) for $30 \mathrm{~min}$, after which $0.9 \mathrm{ml}$ DMEM medium with $2 \%$ FBS was added. After 24 and $48 \mathrm{~h}$ incubation at $37^{\circ} \mathrm{C}$, the virus was titrated in culture supernatants. As shown in Table 3, viral production was similar in both types of cultures.

Reproducibility was studied performing triplicate titrations of A24, O1C and C3 Indaial virus in three passages on OVK (14, 15 and 16), and in three different days (27 replicas). Analysis of the results obtained for different passages, days and repetitions by nested ANOVA showed low variability coefficients that ranged between 4.68 and 0.32 (Table 4). Variability coefficient averages of 1.73 , 1.99 and 1.00 were obtained for subtypes $\mathrm{O}, \mathrm{A}$ and $\mathrm{C}$, respectively.

Table 2. Infection of OVK and $\mathrm{BHK}_{21}$ monolayers grown in 96-well plates after inoculation with different FMDV subtypes. Results are expressed as percentages of positive wells and, in brackets, numbers of positive wells over total numbers of inoculated wells. ${ }^{\#}$ : statistical significance between percentages obtained with $\mathrm{BHK}_{21}$ and $\mathrm{OVK}$, estimated by confidence intervals (ns, ${ }^{* *},{ }^{* * *}$ : non significant, $\mathbf{p}<0.05$ and $\mathrm{p}<0.01$, respectively).

\begin{tabular}{cccc}
\hline Virus & BHK $_{21}$ & OVK & $\mathrm{p}^{\#}$ \\
\hline A2001 & $100(24 / 24)$ & $91.67(44 / 48)$ & $\mathrm{ns}$ \\
A2001 & $34.38(33 / 96)$ & $92.71(89 / 96)$ & ${ }^{* *}$ \\
A2001 & $57.29(55 / 96)$ & $100(96 / 96)$ & ${ }^{* *}$ \\
O1C & $100(96 / 96)$ & $100(48 / 48)$ & $\mathrm{ns}$ \\
O1C & $56.25(36 / 64)$ & $53.12(34 / 64)$ & $\mathrm{ns}$ \\
C3 Indaial & $15.62(10 / 64)$ & $36.45(35 / 96)$ & ${ }^{* * *}$ \\
C3 Indaial & $59.38(57 / 96)$ & $83.33(80 / 96)$ & ${ }^{* * *}$ \\
\hline
\end{tabular}

Table 3. Production of A2001, O1C and C3 Indaial subtypes, after 24 and $48 \mathrm{~h}$ of culture in OVK and $\mathrm{BHK}_{21}$ cells. Results are expressed as TCID $50 / \mathrm{ml}$.

\begin{tabular}{cccccc}
\hline & & \multicolumn{4}{c}{ Virus passage in } \\
\cline { 3 - 6 } & & 24 & 48 & 24 & 48 \\
\hline Titrated in: & & OVK & \multicolumn{3}{c}{ BHK $_{21}$} \\
BHK $_{21}$ & A2001 & 5.33 & 6 & 5.5 & 6 \\
OVK & A2001 & 5.3 & 6.5 & 5.3 & 6.5 \\
BHK $_{21}$ & O1C & 5.66 & 5.33 & 6.00 & 6.33 \\
OVK & O1C & 6.50 & 5.33 & 6.50 & 6.30 \\
BHK $_{21}$ & C3 Indaial & $\leq 0.5$ & 6.15 & $\leq 0.5$ & 5.75 \\
OVK & C3 Indaial & $\leq 0.5$ & 6.5 & $\leq 0.5$ & 6.64 \\
\hline
\end{tabular}

Table 4. Reproducibility of FMDV culturing in OVK, expressed as variation coefficients obtained for FMDV subtypes O1C (O), A2001 (A) and C3 Indaial (C) in different OVK passages, days or repeats. "Mean titer obtained for each viral type expressed as $\log 10$ of TCID50/ml. ${ }^{* *} 95 \%$ confidence interval of mean titers, estimated between different passages and days.

\begin{tabular}{ccccccccc}
\hline & \multicolumn{3}{c}{$\begin{array}{c}\text { Variation coefficient } \\
\text { for OVK }\end{array}$} & \multicolumn{3}{c}{ Viral titer in cell cultures } \\
\hline $\begin{array}{c}\text { FMDV } \\
\text { subtype }\end{array}$ & $\begin{array}{c}\text { Passage } \\
\text { O Day }\end{array}$ & Repeat & OVK $^{*}$ & IC95\% $^{* *}$ & BHK $_{21}$ & IC95\% \\
\hline $\mathrm{O}$ & 4.68 & 1.32 & 1.83 & 4.42 & $(4.27-4.58)$ & 4.58 & $(4.47-4.69)$ \\
$\mathrm{A}$ & 2.55 & 3.12 & 1.02 & 7.22 & $(6.94-7.51)$ & 7.33 & $(6.83-7.33)$ \\
$\mathrm{C}$ & 2.20 & 1.19 & 0.32 & 7.51 & $(7.36-7.67)$ & 7.46 & $(7.42-7.50)$ \\
\hline
\end{tabular}

\section{Conclusion}

In conclusion, our study shows that the OVK cell line is suitable for FMDV sensitive detection and production, showing reproducible results and performance levels that are similar or superior to those obtained with $\mathrm{BHK}_{21}$ cells. Importantly, this system originates from the natural FMDV host, and conserves its viral cell receptors $[7,8]$. Establishment of an OVK bank, stored in liquid nitrogen, allows the immediate application of this system for an early FMDV detection. This type of rapidly available tools is of critical importance in control and eradication campaigns in countries that keep a "free with vaccination" FMDV status, where the virus might be able to spread with mild or subclinical infections.

\section{Acknowledgements}

Financial support from the Argentine Ministry of Science and Technology, MINCyT (PAE-2007-00013), the National Institute of Agricultural Technology, INTA (AESA 201721-PE Fiebre Aftosa) and Universidad del Salvador, Argentina, is gratefully acknowledged.

\section{REFERENCES}

[1] OIE, "Foot and Mouth Disease," In: Manual of Diagnostic Tests and Vaccines for Terrestrial Animals, Chapter 2.1.5, OIE, 2012.

[2] J. B. Clarke and R. E. Spier, "Variation in the Susceptibility of BHK Populations and Cloned Cell Lines to Three Strains of Foot-and-Mouth Disease Virus," Archives of Virology, Vol. 63, No. 1, 1980, pp. 1-9. doi:10.1007/BF01320756

[3] M. P. De Castro, "An Infectious Agent Causing 'Spontaneous' Degeneration of Swine Cells in Vitro," In Vitro, Vol. 9, No. 1, 1973, pp. 8-16. doi:10.1007/BF02615982

[4] N. P. Ferris, G. H. Hutchings, H. J. Moulsdale, J. Golding and J. B. Clarke, "Sensitivity of Primary Cells Immortalised by Oncogene Transfection for the Detection and Iso- 
lation of Foot-and-Mouth Disease and Swine Vesicular Disease Viruses," Veterinary Microbiology, Vol. 84, No. 4, 2002, pp. 307-316. doi:10.1016/S0378-1135(01)00469-2

[5] N. P. Ferris, D. P. King, S. M. Reid, G. H. Hutchings, A. E. Shaw, D. J. Paton, N. Goris, B. Haas, B. Hoffmann, E. Brocchi, et al., "Foot-and-Mouth Disease Virus: A First Inter-Laboratory Comparison Trial to Evaluate Virus Isolation and RT-PCR Detection Methods," Veterinary Microbiology, Vol. 117, No. 2-4, 2006, pp. 130-140. doi:10.1016/j.vetmic.2006.06.001
[6] L. J. M. Reed, "A Simple Method of Estimating Fifty Percent Endpoints," The American Journal of Hygiene, Vol. 27, 1938, pp. 493-497.

[7] T. Jackson, A. P. Mould, D. Sheppard and A. M. King, "Integrin Alphavbeta1 Is a Receptor for Foot-and-Mouth Disease Virus," Journal of Virology, Vol. 76, No. 3, 2002, pp. 935-941. doi:10.1128/JVI.76.3.935-941.2002

[8] T. Jackson, A. M. King, D. I. Stuart and E. Fry, "Structure and Receptor Binding," Virus Research, Vol. 91, No. 1, 2003, pp. 33-46. doi:10.1016/S0168-1702(02)00258-7 